\title{
Interleukin-17A-Induced Epithelial-Mesenchymal Transition of Human Intrahepatic Biliary Epithelial Cells: Implications for Primary Biliary Cirrhosis
}

\author{
Qingshui Huang, ${ }^{1,2, *}$ Shuai Chu, ${ }^{1, *}$ Xiaofeng Yin, ${ }^{1, *}$ Xiaobin Yu, ${ }^{1}$ Chunmin Kang, ${ }^{1}$ \\ Xin $\mathrm{Li}^{1}$ and Yurong Qiu ${ }^{1}$
}

${ }^{1}$ Laboratory Medicine Center, Nanfang Hospital, Southern Medical University, Guangzhou, China

${ }^{2}$ Clinical Laboratory, First Affiliated Hospital of Nanchang University, Nanchang, China

\begin{abstract}
Primary biliary cirrhosis (PBC) is an autoimmune chronic liver disease with worldwide increasing morbidity. However, the etiology of PBC is still unclear. Recently, the epithelial-mesenchymal transition (EMT) and interleukin-17A (IL-17A), a pro-inflammatory cytokine, were proposed to be involved in the pathogenesis of PBC. Therefore, in this study, we aimed to clarify the roles of IL-17A and/or EMT in the onset of PBC. The results showed that the median serum IL-17A level was significantly higher in 29 PBC patients (average course of 40.69 months) than that of 11 healthy controls. The intrahepatic biliary epithelial cells (IBECs), the major target of destruction in PBC, underwent EMT in PBC patients. The immunohistochemical analysis revealed that the protein levels of IL-17A receptor were increased in IBECs and the IL-17A protein was accumulated around the IBECs in the PBC patients. These results imply that the IL-17A-mediated signaling and EMT of intrahepatic biliary epithelial cells (IBEC-EMT) are key pathogenic processes of PBC. To study the association between IL-17A and IBECs-EMT, we then examined if IL-17A induced EMT using a human cell line of IBECs (HIBECs). After the treatment with IL-17A for $48 \mathrm{~h}$, HIBECs changed into bipolar cells with a fibroblastic morphology. Additionally, the results of real-time PCR and Western blot analyses demonstrated that IL-17A up-regulated the expression of a mesenchymal marker vimentin and downregulated the expression of an epithelial marker E-cadherin in HIBECs in the dose- and time-dependent manners. These results suggest that IL-17A may play an important role in the IBECs-EMT.
\end{abstract}

Keywords: epithelial-mesenchymal transition; interleukin-17A; intrahepatic biliary epithelial cells; primary biliary cirrhosis; progressive ductopenia

Tohoku J. Exp. Med., 2016 December, 240 (4), 269-275. C (c) 2016 Tohoku University Medical Press

\section{Introduction}

Primary biliary cirrhosis (PBC) is an autoimmune chronic liver disease characterized by the progressive destruction of intrahepatic bile ducts, leading to cholestasis, cirrhosis, and liver failure (Isse et al. 2006; Chan et al. 2014). Epidemiological studies have revealed increased morbidity of PBC in recent years (Kim et al. 2000; Sood et al. 2004; Peters et al. 2007; Zhang et al. 2015a). Additionally, diagnosed $\mathrm{PBC}$ patients have been increased in China (Zhang et al. 2015b). At present, the etiology of $\mathrm{PBC}$ is still unclear. Several studies have suggested involvement of multiple factors such as genetic predisposition, environmental factors, viral and bacterial infections, autoantibodies, dysregulated immune functions, chemokines, antigen-presenting cells, autophagy, aging, and apoptosis (Jones 2008; Prince et al. 2010; Berg 2011; Liu et al. 2012; Zhang et al. 2015a).
PBC is marked by the destruction of intrahepatic bile ducts and loss of intrahepatic biliary epithelial cells (IBECs), which underpins most of the subsequent cholestatic damages (Jones 2008). The mechanisms that trigger IBECs loss in PBC have been subjected to intensive studies (Kaplan and Gershwin 2005; Lindor et al. 2009). One potential mechanism for IBECs loss is epithelial to mesenchymal transition (EMT), in which epithelial cells undergo phenotypic reprogram, leading to loss of epithelial markers and properties but gain of features related to fibroblasts (Robertson et al. 2007).

EMT has been found to contribute to fibrosis in multiple pathological conditions and to be regulated by cytokines and other pro-inflammatory mediators (Borthwick et al. 2013). Interleukin-17 (IL-17A), a typical pro-inflammatory cytokine, has recently been shown to induce EMT of alveolar epithelial cells (Mi et al. 2011) and bronchial epithelial cells (Ji et al. 2013; Vittal et al. 2013). Interestingly, fre-

Received August 1, 2016; revised and accepted November 9, 2016. Published online December 3, 2016; doi: 10.1620/tjem.240.269.

*These authors contributed equally to this work.

Correspondence: Yurong Qiu, Laboratory Medicine Center, Nanfang Hospital, Southern Medical University, Guangzhou 510515, China.

e-mail: qiuyuronggz@126.com 
quencies of IL-17A -positive lymphocytic cells are increased in the liver of PBC patients (Lan et al. 2009) and IL-17A expression is elevated in peripheral blood of PBC patients (Qian et al. 2013). However, the association between IL-17A and IBECs-EMT and their roles in the pathogenesis of $\mathrm{PBC}$ remain unclear.

In this study, we investigated if IBECs underwent EMT in PBC patients and evaluated the IL-17A response in this process. To further study the association between IL-17A and IBEC-EMT, we examined if IL-17A induced EMT of human IBECs in vitro. Our study sheds light on the association between IL-17A and IBECs-EMT and provides new understanding of the pathogenesis of PBC.

\section{Materials and Methods}

\section{Patients}

PBC patients were diagnosed according to clinical manifestation, elevated serum alkaline phosphatase level, seropositivity for anti-mitochondrial antibody and diagnostic liver histology as described previously (Carey et al. 2015). PBC was categorized into four phases based on the following histological changes: stage I, cholangiolitis; stage II, bile duct proliferation; stage III, cicatrization; and stage IV, liver cirrhosis (Ludwig et al. 1978). Serum samples were obtained from 29 PBC patients ( 2 males and 27 females; 19-80 years of age, mean age: 51 years, course of disease: 10 day to 315 month, average course: 40.69 months), and 11 healthy volunteers (2 males and 9 females; 29-64 years of age, mean age: 46.8 years) from July 2014 to September 2015. For histological and immunohistochemical analysis, liver biopsies were obtained from 9 PBC patients ( 1 male and 8 females; 28-65 years of age, mean age: 51 years; 3 in stage I, 4 in stage II, 2 in stage III) and 4 healthy volunteers who underwent acute liver rupture caused by trauma and received emergency surgical resection of the damaged liver tissues ( 3 males and 1 females; 34-58 years of age, mean age: 47 years) from January 2006 to July 2015. Samples were collected from patients before treatment. All the patients and healthy volunteers were recruited from the Nanfang Hospital of Southern Medical University, and the First Affiliated Hospital of Nanchang University. All samples were collected from patients and healthy volunteers after obtaining informed consent in accordance with a protocol approved by the Ethics Committee of Nangfang Hospital, Southern Medical University (Guangzhou, China) and the First Affiliated Hospital of Nanchang University (Nanchang, China).

\section{Enzyme-linked Immunosorbent Assay (ELISA) for IL-17A in serum}

Serum IL-17A was detected by ELISA using the human IL-17A Platinum ELISA kit (eBioscience, Inc, San Diego, CA, USA) according to the manufacturer's instructions.

\section{Histology and immunohistochemistry}

Liver biopsies were fixed with $4 \%$ paraformaldehyde, embedded in paraffin, and cut into $3 \mu \mathrm{m}$-thin sections. Tissue thin sections were stained with hematoxylin and eosin (H\&E) and histological images were acquired using a Leica DM2500 Upright Microscope as previously described (Yang et al. 2009). For immunohistochemical analysis, liver thin-sections $(4 \mu \mathrm{m})$ were immunostained using the standard microwave protocol and imaged with a Leica DM2500 Upright Microscope as described previously (Kumada et al. 2004).
Antibodies include a mouse monoclonal antibody against IL-17A (4K5F6, Abcam, Cambridge, MA, USA; 1:100 dilution), an anti-IL17A receptor antibody (Abcam; 1:100 dilution), a rabbit monoclonal antibody against E-cadherin (24E10, Cell Signaling Technology, Inc, Danvers, MA, USA; 1:100 dilution), and a rabbit monoclonal antibody against vimentin (D21H3, Cell Signaling Technology, Inc; 1:100 dilution). Additionally, antibodies of cytokeratin 7 and 19 (Fuzhou Maixin Biotech. Co., Ltd., China; 1: 100 dilution), two markers for IBECs, were used for identification of IBECs in liver tissues.

\section{Cell culture}

A human cell line of IBEC (HIBECs), derived from the normal liver, was obtained from the cell bank of Guangzhou Jennio Biotech Co., Ltd. (Guangzhou, GD, China) and were cultured in RPMI1640 medium (Invitrogen, Carlsbad, CA, USA) supplemented with $10 \mathrm{ng} /$ $\mathrm{ml}$ epidermal growth factor (Invitrogen) and 10\% fetal bovine serum (Thermo Fisher Scientific, Waltham, MA, USA) at $37^{\circ} \mathrm{C}$ in a $5 \% \mathrm{CO}_{2}$ humidified atmosphere. HIBECs were stimulated with recombinant human IL-17A (R\&D Systems, Inc.) at the concentrations of 10, 50 and $100 \mathrm{ng} / \mathrm{ml}$ for 24,48 and $72 \mathrm{~h}$ to induce EMT and were examined using a Leica DM2500 Upright Microscope.

\section{Quantitative real-time $R T-P C R$}

Total RNA was extracted from HIBECs using a TRIzol Reagent (Takara, Dalian, China) according to the manufacturer's instruction and was reversely transcribed into the first-strand cDNA using the PrimeScript RT reagent kit (Takara). Each $20 \mu \mathrm{L}$ PCR mixture contained $1 \mu \mathrm{L}$ of cDNA, $10 \mu \mathrm{L}$ of SYBR Green PCR Master Mix (Takara), and $0.5 \mathrm{pM}$ of each primer (E-cadherin: 5'-TACACT GCCCAGGAGCCAGA-3' and 5'- TGGCACCAGTGTCCG GATTA-3'; Vimentin: 5'-GAGAACTTTGCCGTTGAAGC-3' and 5'-GCTTCCTGTAGGTGGCAATC-3'; glyceraldehyde-3-phosphate dehydrogenase (GAPDH): 5'-AGAAGGCTGGGGCTCATTTG-3' and 5'-AGGGGCCATCCACAGTCTTC-3'). Quantitative real-time PCR was carried out using the ABI 7500 Fast Real Time PCR system (Applied Biosystems, Foster City, CA, USA). PCR conditions used were $50^{\circ} \mathrm{C}$ for $2 \mathrm{~min}, 95^{\circ} \mathrm{C}$ for $2 \mathrm{~min}$, followed by 40 cycles of $15 \mathrm{~s}$ at $95^{\circ} \mathrm{C}$ and $60 \mathrm{~s}$ at $60^{\circ} \mathrm{C}$. The $\mathrm{Ct}$ values of an internal control (GAPDH) and the target genes were determined. The $2^{-\triangle \Delta \mathrm{Ct}}$ method was used to calculate the relative expression. The transcript levels of examined genes were quantitatively normalized to GAPDH.

\section{Western blot analysis}

Total proteins were extracted from HIBECs with a complete cell-lysis buffer (Keygen Biotech, Jiangsu, China), with newly added protease and phosphatase inhibitors. Protein concentrations were quantified using the bicinchoninic acid protein assay kit (KeyGen Biotechnologies, Nanjing, China). Aliquots of $50 \mu \mathrm{g}$ proteins were separated by $10 \%$ SDS-polyacrylamide gel electrophoresis and transferred to polyvinylidene fluoride membranes (GE Healthcare, Little Chalfont, UK). Following the transfer, the membranes were blocked with $5 \%$ bovine serum albumin for $1 \mathrm{~h}$ at room temperature, then incubated with primary antibodies including a rabbit monoclonal antibody against E-cadherin (24E10, Cell Signaling Technology; 1:1,000 dilution), a rabbit monoclonal antibody against Vimentin (D21H3, Cell Signaling Technology, Inc; 1:1,000 dilution), or a rabbit monoclonal antibody against $\beta$-Actin (13E5, Cell Signaling Technology, Inc; $1: 1,000$ dilution) overnight at $4^{\circ} \mathrm{C}$, and incubated with an HRPconjugated goat anti-rabbit IgG secondary antibody (Bioss, Beijing, 
China) for $1 \mathrm{~h}$ at room temperature. After incubation with the secondary antibody, membranes were visualized using enhanced chemiluminescent (ECL) detection method (ECL Plus Western Blotting Detection System; Amersham Biosciences, Foster City, CA, USA) according to the manufacturer's instructions.

\section{Statistical analysis}

Data are expressed as the mean \pm standard error (SEM). Statistical analysis was performed using one-way analysis of variance (ANOVA), followed by post hoc Newman-Keuls test for multiple comparisons. For comparison of two groups, data were analyzed by the Student's $t$-test. All data were analyzed using the software SPSS 13.0 (Statistical Package for Social Sciences; SPSS, Munich, Germany). Statistically significant differences were determined at $P$ $<0.05$.

\section{Results}

\section{IBECS-EMT in livers of PBC patients}

A previous case report has revealed IBECs-EMT in transplanted liver tissues of a PBC patient, suggesting that IBECs-EMT might occur at early stage of PBC (Robertson et al. 2007). However, it has been unclear if IBECs-EMT occurs in primary PBC patients. In this study, H\&E staining of liver thin sections revealed obvious lymphocyte and macrophage infiltration around and within the bile ducts in PBC livers compared with healthy controls (Fig. 1A, B). The liver pathology in the patient (Fig. 1B) was stage I PBC according to the histological changes described in the previous report (Ludwig et al. 1978). Immunohistochemical staining showed that the liver tissues from PBC patients contained increased number of vimentin (mesen-
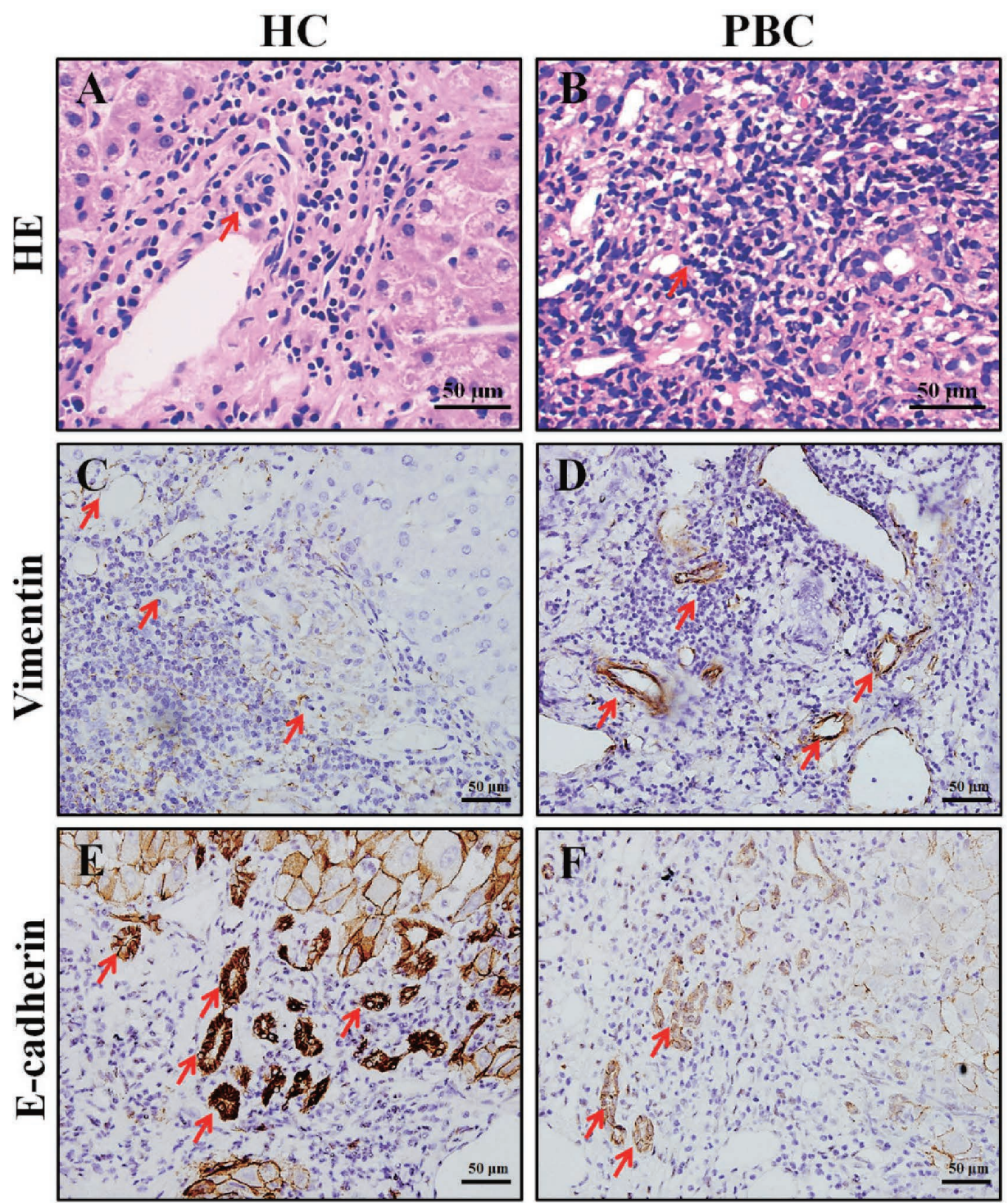

Fig. 1. Intrahepatic EMT in PBC patients.

A, B, H\&E staining of liver thin-sections from a healthy control and PBC patient. C-F, Immunohistochemical detection of Vimentin (C and D, a mesenchymal marker) and E-cadherin (E and F, an epithelial marker). Red arrows indicated bile ducts. Data shown are representative of 3 experiments from 3 patients at stage I. 
chymal marker)-positive IBECs (Fig. 1C, D) and decreased number of E-cadherin (epithelial marker)-positive IBECs (Fig. 1E, F), compared to healthy controls. Moreover, vimentin-positive cells in $\mathrm{PBC}$ patients appeared to express higher levels of vimentin than those in healthy controls, while E-cadhein-positive cells in $\mathrm{PBC}$ patients expressed lower levels of this molecule than those in healthy controls (Fig. 1C-F). These phenotypes were significant in the 3 stage I PBC patients and less obvious in the 4 stage II and 2 stage III PBC patients (data not shown). These results suggest that IBECs-EMT occurs in primary PBC patients.

\section{Elevated IL-17A and IL-17A receptor in the livers of PBC patients}

Previous studies showed that frequencies of IL-17Apositive lymphocytic cells were increased in the liver from PBC patients (Lan et al. 2009) and IL-17A expression was elevated in peripheral blood of PBC patients (Qian et al. 2013). Additionally, IL-17A induced EMT of epithelial cells (Mi et al. 2011; Ji et al. 2013; Vittal et al. 2013). Therefore, to explore the association between IL-17A and IBECs-EMT, we first compared serum IL-17A concentrations between healthy controls and $\mathrm{PBC}$ patients. The median serum IL-17A levels in healthy controls and PBC patients were $1.89 \pm 0.52$ and $2.63 \pm 1.27 \mathrm{pg} / \mathrm{ml}$, respectively (Table 1). The differences between healthy control and PBC patients were statistically significant $(P<0.05)$. Thus, IL-17A was elevated in PBC patients compared with healthy controls. Our data confirmed similar findings in previous studies in PBC patients (Lan et al. 2009; Qian et al. 2013).

We further examined whether IL-17A levels were increased in the liver of PBC patients by immunohistochemistry. We detected IL-17A- (IL-17A ${ }^{+}$) and IL-17A receptor-positive cells (IL-17 $\mathrm{R}^{+}$) near portal tracts and around bile ducts and these IL-17A $\mathrm{A}^{+}$or IL- $17 \mathrm{R}^{+}$cells were markedly increased in the liver from $\mathrm{PBC}$ patients compared with healthy controls. Additionally, the protein levels of IL-17A receptor were increased in IBECs and the IL-17A proteins were accumulated around the IBECs in the PBC patients (Fig. 2A, B). We found that 7 of the 9 examined PBC patients had increased IL-17A and IL-17R protein levels in livers.
The impact of IL-17A on the morphological changes of HIBECS in vitro

Increased IL-17A $\mathrm{A}^{+}$and $\mathrm{IL}-17 \mathrm{R}^{+}$cells in the liver of $\mathrm{PBC}$ patients prompted us to investigate if IL-17A was involved in EMT. During in vitro culture in the absence of IL-17A, HIBECs displayed a classic cobble-stone shape. After stimulated with recombinant human IL-17A at 10, 50 and $100 \mathrm{ng} / \mathrm{ml}$ for $48 \mathrm{~h}$, HIBECs displayed a dose-dependent transition to a spindle-shape, fibroblast-like morphology (Fig. 3A). Treatment of HIBECs with $50 \mathrm{ng} / \mathrm{ml}$ of IL-17A for different times revealed a time-dependent evolution of fibroblast-like morphological changes that started at 24 hours and exacerbated after 72 hours of treatment (Fig. 3). Thus, IL-17A appeared to be able to induce HIBECs to undergo fibroblast-like morphological change in vitro.

\section{The induction of HIBECs-EMT by IL-17A in vitro}

The ability of IL-17A to induce a fibroblast-like morphology of HIBESs suggested the possibility that it might trigger EMT of HIBECs. We further determined if IL-17A treatment might affect vimentin and E-cadherin expression in cultured HIBECs. As shown in Fig. 4A, B, vimentin mRNA was increased while E-cadherin mRNA was decreased in dose- and time-dependent manners following IL-17A treatment. Correlated with altered mRNA levels, vimentin protein and E-cadherin protein levels were also increased and decreased, respectively, following IL-17A treatment (Fig. 4C, D). These results together with those shown in Fig. 3 suggested that IL-17A could induce HIBECs-EMT in vitro.

\section{Discussion}

PBC has been regarded as a landmark disease for study the pathophysiology of conditions in which autoimmune phenomena is prominent, as the presence of serum autoantibodies and autoantigens for $\mathrm{PBC}$ was recognized (Mackay 1958; Yeaman et al. 1988). However, the pathogenesis of PBC remains unclear, owing in large part to increasingly contradictory observations made in recent years. Ultimately, any model for PBC pathogenesis must be able to explain IBECs loss and the development of progressive ductopenia. Initial researches suggested that apoptosis was an important mechanism for IBECs loss in livers of PBC (Kuroki et al. 1996; Harada et al. 1997; Tinmouth et al. 2002; Kawata et al. 2012). Nevertheless, the apoptosis of IBECs is thought to be predominant in the middle

Table 1. Serum IL-17A levels in PBC patients.

\begin{tabular}{lllll}
\hline groups & male & female & Age (years) & IL-17A $(\mathrm{pg} / \mathrm{ml})$ \\
\hline PBC & 2 & 27 & $51.5 \pm 14.2$ & $2.63 \pm 1.27^{*}$ \\
Health control & 2 & 9 & $46.9 \pm 12.5$ & $1.89 \pm 0.52$ \\
\hline
\end{tabular}

Data shown represent mean \pm SEM. $* P<0.05$ determined by Student's $t$-test. 


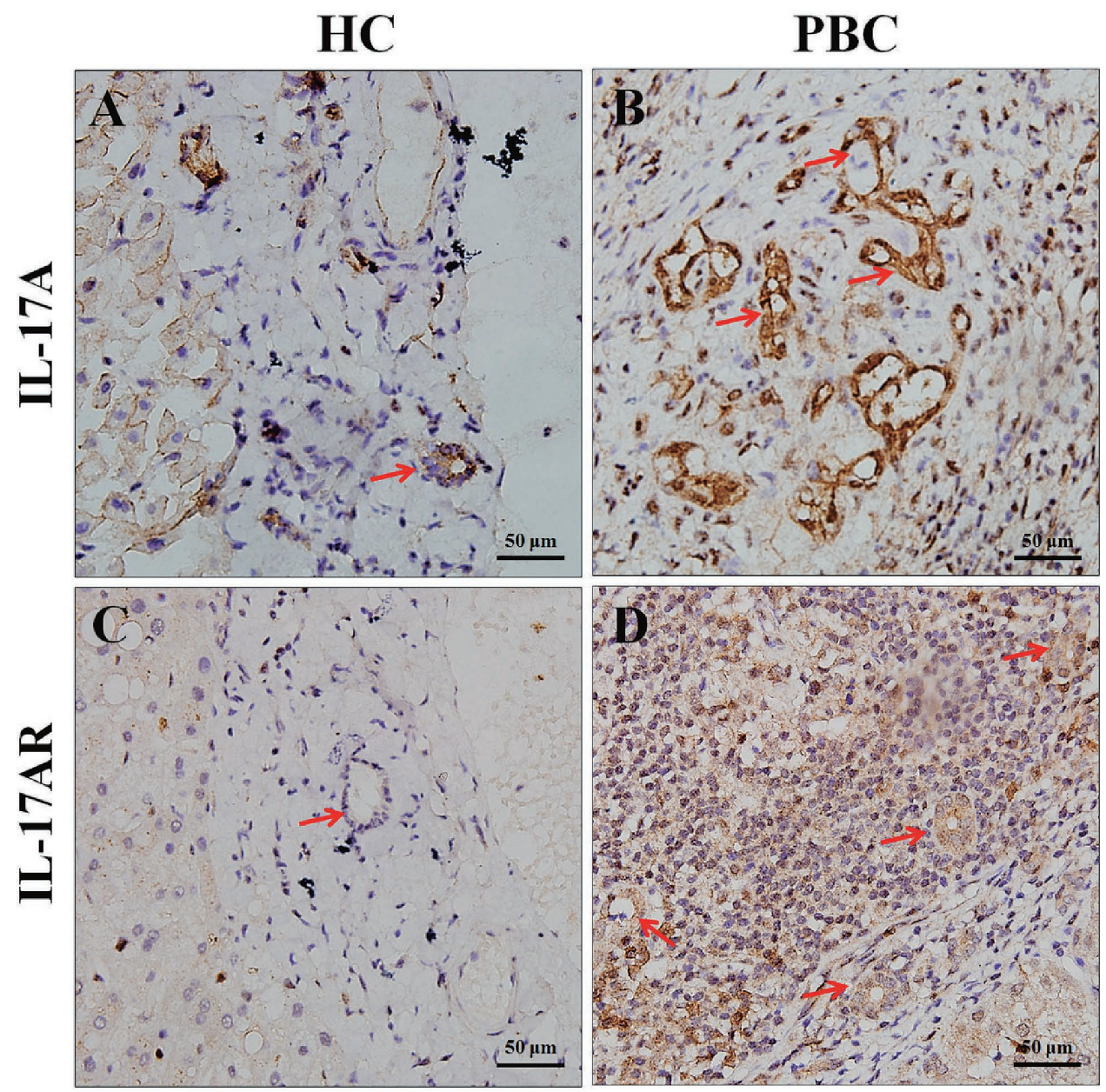

Fig. 2. Increased hepatic expression of IL-17A and IL-17AR in PBC patients.

Liver thin sections of tissue biopsies from healthy controls (HC) and Stage II of PBC patients (PBC) were used for immunohistochemical analysis of IL17A (A and B) and IL-17A receptor (IL17AR, C and D). Red arrows indicated the bile ducts. Data shown are representative of 4 experiments from 4 patients at stage II.

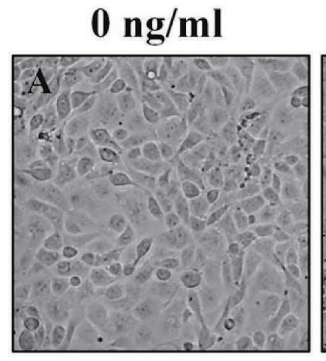

$0 \mathbf{h}$

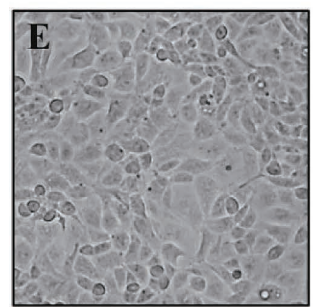

$10 \mathrm{ng} / \mathrm{ml}$

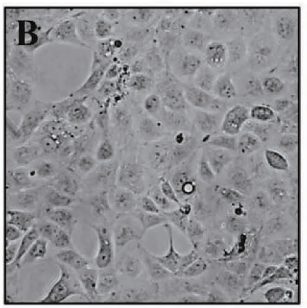

$24 \mathrm{~h}$

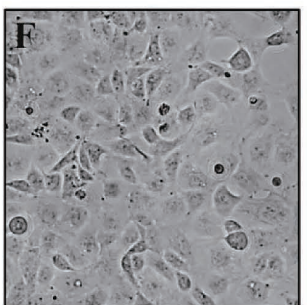

$50 \mathrm{ng} / \mathrm{ml}$

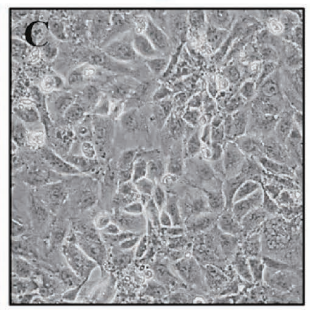

$48 \mathrm{~h}$

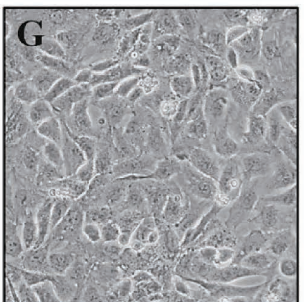

$100 \mathrm{ng} / \mathrm{ml}$

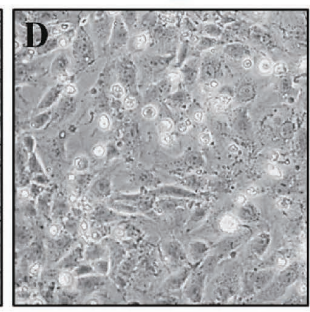

$72 \mathrm{~h}$

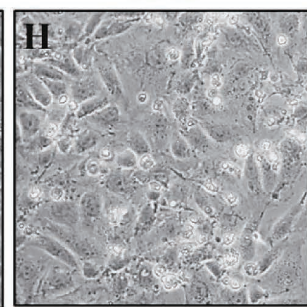

Fig. 3. IL-17A induces morphological change of HIBECs.

A-D, HIBECs were unstimulated or stimulated with 10, 50, or $100 \mathrm{ng} / \mathrm{ml}$ IL17A for $48 \mathrm{~h}$. E-H, HIBECs were unstimulated or stimulated with $50 \mathrm{ng} / \mathrm{ml}$ IL17A 24, 48 and $72 \mathrm{hrs}$. Cell morphology was examined with a light microscope with $200 \times$ magnification. 

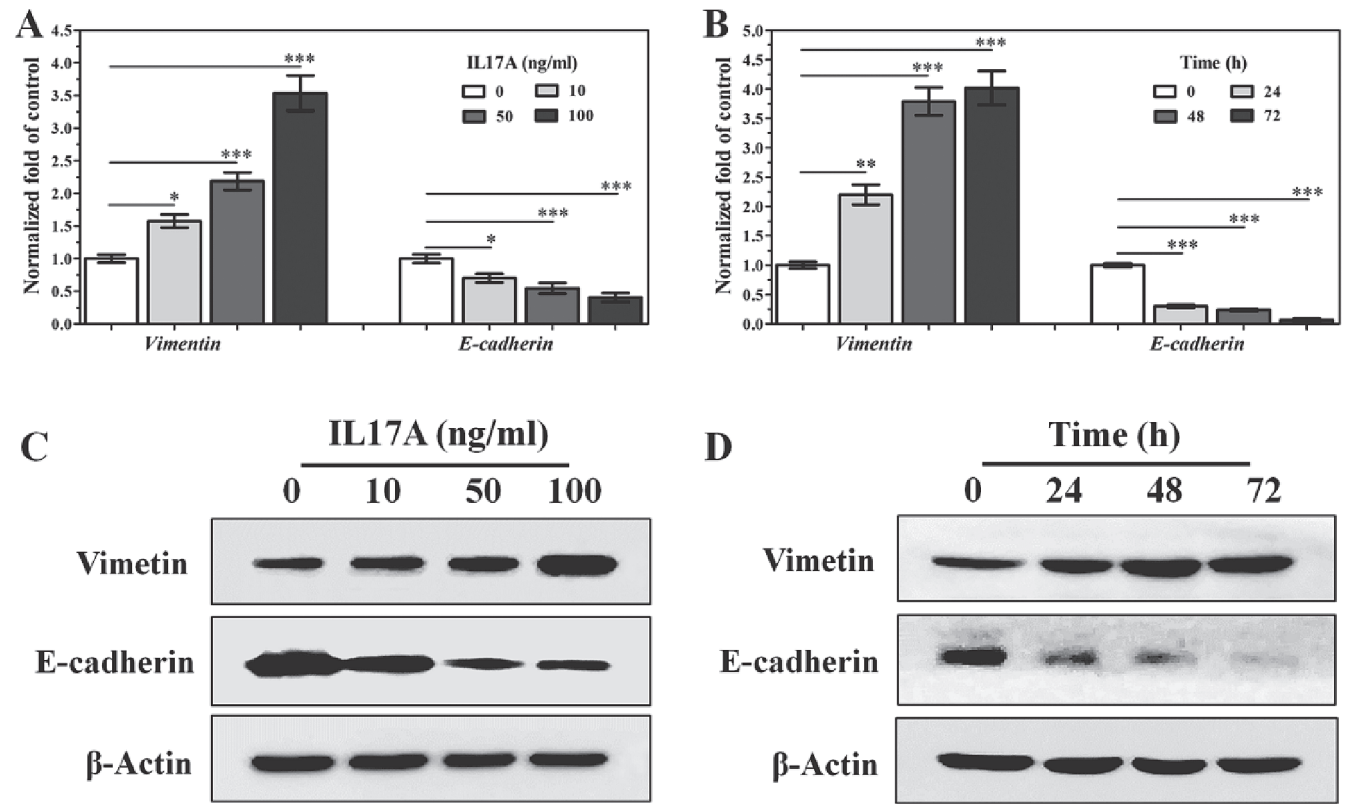

D

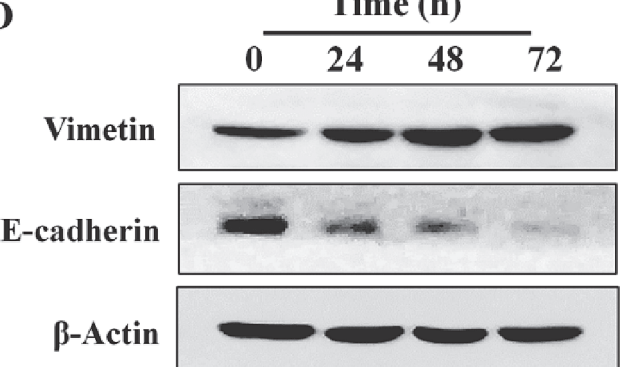

Fig. 4. Elevated expression of Vimentin and decreased expression of E-cadherin in cultured HIBECs following IL-17A treatment.

HIBECs were similarly cultured and stimulated with IL-17A as in Fig. 3. At indicated doses or time points, total RNAs were isolated for quantitative real-time RT-PCR (A, B) and cell lysates were made for immunoblotting analysis (C, D). A. Relative mRNA levels of vimentin and E-cadherin in response to different concentrations of IL-17A. B. Relative mRNA levels of vimentin and E-cadherin after IL-17A treatment for the indicated times. Data are expressed as the mean \pm SEM. $* P<0.05, * * P<0.01$ and $* * * P<0.001$. C. Vimentin and E-cadherin protein levels detected by immunoblotting analysis in response to different concentrations of IL-17A. D. Vimentin and E-cadherin protein levels detected by immunoblotting analysis after IL-17A treatment for the indicated times. Data shown are representative of 3 experiments.

stages (II-III) of PBC and may be relatively infrequent in the earlier stages (Robertson et al. 2007; Jones 2008). The previous study from a $\mathrm{PBC}$-recurred patient after liver transplantation revealed that HIBECs-EMT may predominate in very early process of PBC (Robertson et al. 2007). Thus in this study, we examined if IBECs underwent EMT in livers of patients at the early stage of PBC. In sequential liver biopsies, we demonstrated increased expression of Vimentin (a mesenchymal marker) and decreased expression of E-cadherin (epithelial marker) within IBECs of stage I PBC patients (Fig. 1). These results indicate that these cells are undergoing EMT and suggest that this phenotypic shift may explain the "loss" of IBEC and "gain" of progressive ductopenia, key characters of PBC.

In this study, we found that serum IL-17A level was increased (Table 1) in PBC patients. IL-17A-positive cells around the intrahepatic bile ducts were higher in the early stages of $\mathrm{PBC}$ compare to the healthy controls and IL-17A proteins were accumulated around the IBECs in the PBC patients (Fig. 2A, B). These results suggest that IL-17A in PBC exerts pro-inflammatory effects in early stage. These results are consistent with the previous results that the expression of IL-17A was increased in PBC patients (Lan et al. 2009; Qian et al. 2013). We also found the elevated expression of IL-17A receptor in IBECs in PBC patients (Fig. 2C, D), suggesting the IL-17A-mediated signaling in response to $\mathrm{PBC}$. Although these results all indicated that
IBECs-EMT and IL-17A-mediated signaling are key pathogenetic processes in the early stage of $\mathrm{PBC}$, the association between IBECs-EMT and IL-17A is not clear. Thus we investigated if IL-17A was involved in EMT. IL-17A appeared to be able to induce HIBECs to undergo fibroblast-like morphological change in vitro (Fig. 3) and increased the expression of Vimentin mRNA and decreased the expression of E-cadherin mRNA in dose- and timedependent manners (Fig. 4). These results indicate that IL-17A induces HIBECs-EMT in vitro, consistent with the results that IL-17A can also induce EMT in alveolar and bronchial epithelial cells (Mi et al. 2011; Ji et al. 2013; Vittal et al. 2013). These results indicated that IL-17A played a potential role in inducing EMT of IBECs and subsequent peribiliary fibrosis.

In conclusion, the present study confirmed the previous studies that the IBECs-EMT and IL-17A-mediated signaling are key pathogenetic processes in the early stage of PBC. This study also found that IL-17A induced the EMT of HIBECs in vitro. Our study suggests a critical mechanism underlying the pathogenesis of PBC.

\section{Acknowledgments}

We thank Prof. Xiaoping Zhong (Department of Immunology, Duke University Medical Center) for suggestion and revision of the manuscript. This work was supported by Natural Science Foundation of Jiangxi, China (No. 20161BAB205248) 
and the project of Health and Family Planning Commission of Jiangxi province (No. 20155119) for Q.S. Huang. This work was also granted by Guangdong Natural Science Foundation (No. 2016A030313525) and Science and Technology Program of Guangzhou, China (No. 201607010015) for Y.R. Qiu.

\section{Conflict of Interest}

The authors declare no conflict of interest.

\section{References}

Berg, P.A. (2011) The role of the innate immune recognition system in the pathogenesis of primary biliary cirrhosis: a conceptual view. Liver Int., 31, 920-931.

Borthwick, L.A., Wynn, T.A. \& Fisher, A.J. (2013) Cytokine mediated tissue fibrosis. Biochim. Biophys. Acta, 1832, 10491060 .

Carey, E.J., Ali, A.H. \& Lindor, K.D. (2015) Primary biliary cirrhosis. Lancet, 386, 1565-1575.

Chan, A.W., Chan, R.C., Wong, G.L., Wong, V.W., Choi, P.C., Chan, H.L. \& To, K.F. (2014) Evaluation of histological staging systems for primary biliary cirrhosis: correlation with clinical and biochemical factors and significance of pathological parameters in prognostication. Histopathology, $\mathbf{6 5}$, 174-186.

Harada, K., Ozaki, S., Gershwin, M.E. \& Nakanuma, Y. (1997) Enhanced apoptosis relates to bile duct loss in primary biliary cirrhosis. Hepatology, 26, 1399-1405.

Isse, K., Harada, K., Sato, Y. \& Nakanuma, Y. (2006) Characterization of biliary intra-epithelial lymphocytes at different anatomical levels of intrahepatic bile ducts under normal and pathological conditions: Numbers of CD4+CD28- intraepithelial lymphocytes are increased in primary biliary cirrhosis. Pathol. Int., 56, 17-24.

Ji, X., Li, J., Xu, L., Wang, W., Luo, M., Luo, S., Ma, L., Li, K., Gong, S., He, L., Zhang, Z., Yang, P., Zhou, Z., Xiang, X. \& Wang, C.Y. (2013) IL4 and IL-17A provide a Th2/ Th17-polarized inflammatory milieu in favor of TGF-beta1 to induce bronchial epithelial-mesenchymal transition (EMT). Int. J. Clin. Exp. Pathol., 6, 1481-1492.

Jones, D.E. (2008) Pathogenesis of primary biliary cirrhosis. Clin. Liver Dis., 12, 305-321.

Kaplan, M.M. \& Gershwin, M.E. (2005) Primary Biliary Cirrhosis. N. Engl. J. Med., 353, 1261-1273.

Kawata, K., Kobayashi, Y., Gershwin, M.E. \& Bowlus, C.L. (2012) The immunophysiology and apoptosis of biliary epithelial cells: primary biliary cirrhosis and primary sclerosing cholangitis. Clin. Rev. Allergy Immunol., 43, 230-241.

Kim, W.R., Lindor, K.D., Locke, G.R. 3rd, Therneau, T.M., Homburger, H.A., Batts, K.P., Yawn, B.P., Petz, J.L., Melton, L.J. 3rd \& Dickson, E.R. (2000) Epidemiology and natural history of primary biliary cirrhosis in a US community. Gastroenterology, 119, 1631-1636.

Kumada, T., Tsuneyama, K., Hatta, H., Ishizawa, S. \& Takano, Y. (2004) Improved 1-h rapid immunostaining method using intermittent microwave irradiation: practicability based on 5 years application in Toyama Medical and Pharmaceutical University Hospital. Mod. Pathol., 17, 1141-1149.

Kuroki, T., Seki, S., Kawakita, N., Nakatani, K., Hisa, T., Kitada, T. \& Sakaguchi, H. (1996) Expression of antigens related to apoptosis and cell proliferation in chronic nonsuppurative destructive cholangitis in primary biliary cirrhosis. Virchows Arch., 429, 119-129.

Lan, R.Y., Salunga, T.L., Tsuneyama, K., Lian, Z.X., Yang, G.X., Hsu, W., Moritoki, Y., Ansari, A.A., Kemper, C., Price, J., Atkinson, J.P., Coppel, R.L. \& Gershwin, M.E. (2009) Hepatic IL-17 responses in human and murine primary biliary cirrhosis. J. Autoimmun., 32, 43-51.
Lindor, K.D., Gershwin, M.E., Poupon, R., Kaplan, M., Bergasa, N.V. \& Heathcote, E.J.; American Association for Study of Liver Diseases (2009) Primary biliary cirrhosis. Hepatology, 50, 291-308.

Liu, J.Z., Almarri, M.A., Gaffney, D.J., Mells, G.F., Jostins, L., Cordell, H.J., Ducker, S.J., Day, D.B., Heneghan, M.A., Neuberger, J.M., Donaldson, P.T., Bathgate, A.J., Burroughs, A., Davies, M.H., Jones, D.E., et al. (2012) Dense finemapping study identifies new susceptibility loci for primary biliary cirrhosis. Nat. Genet., 44, 1137-1141.

Ludwig, J., Dickson, E.R. \& McDonald, G.S. (1978) Staging of chronic nonsuppurative destructive cholangitis (syndrome of primary biliary cirrhosis). Virchows Arch. A Pathol. Anat. Histol., 379, 103-112.

Mackay, I.R. (1958) Primary biliary cirrhosis showing a high titer of autoantibody; report of a case. N. Engl. J. Med., 258, $185-188$.

Mi, S., Li, Z., Yang, H.Z., Liu, H., Wang, J.P., Ma, Y.G., Wang, X.X., Liu, H.Z., Sun, W. \& Hu, Z.W. (2011) Blocking IL-17A promotes the resolution of pulmonary inflammation and fibrosis via TGF-beta1-dependent and independent mechanisms. J. Immunol., 187, 3003-3014.

Peters, M.G., Di Bisceglie, A.M., Kowdley, K.V., Flye, N.L., Luketic, V.A., Munoz, S.J., Garcia-Tsao, G., Boyer, T.D., Lake, J.R., Bonacini, M. \& Combes, B. (2007) Differences between Caucasian, African American, and Hispanic patients with primary biliary cirrhosis in the United States. Hepatology, 46, 769-775.

Prince, M.I., Ducker, S.J. \& James, O.F. (2010) Case-control studies of risk factors for primary biliary cirrhosis in two United Kingdom populations. Gut, 59, 508-512.

Qian, C., Jiang, T., Zhang, W., Ren, C., Wang, Q., Qin, Q., Chen, J., Deng, A. \& Zhong, R. (2013) Increased IL-23 and IL-17 expression by peripheral blood cells of patients with primary biliary cirrhosis. Cytokine, 64, 172-180.

Robertson, H., Kirby, J.A., Yip, W.W., Jones, D.E. \& Burt, A.D. (2007) Biliary epithelial-mesenchymal transition in posttransplantation recurrence of primary biliary cirrhosis. Hepatology, 45, 977-981.

Sood, S., Gow, P.J., Christie, J.M. \& Angus, P.W. (2004) Epidemiology of primary biliary cirrhosis in Victoria, Australia: high prevalence in migrant populations. Gastroenterology, 127, 470-475.

Tinmouth, J., Lee, M., Wanless, I.R., Tsui, F.W., Inman, R. \& Heathcote, E.J. (2002) Apoptosis of biliary epithelial cells in primary biliary cirrhosis and primary sclerosing cholangitis. Liver, 22, 228-234.

Vittal, R., Fan, L., Greenspan, D.S., Mickler, E.A., Gopalakrishnan, B., Gu, H., Benson, H.L., Zhang, C., Burlingham, W., Cummings, O.W. \& Wilkes, D.S. (2013) IL-17 induces type $\mathrm{V}$ collagen overexpression and EMT via TGF-beta-dependent pathways in obliterative bronchiolitis. Am. J. Physiol. Lung Cell. Mol. Physiol., 304, L401-L414.

Yang, H.Z., Cui, B., Liu, H.Z., Chen, Z.R., Yan, H.M., Hua, F. \& Hu, Z.W. (2009) Targeting TLR2 attenuates pulmonary inflammation and fibrosis by reversion of suppressive immune microenvironment. J. Immunol., 182, 692-702.

Yeaman, S.J., Fussey, S.P., Danner, D.J., James, O.F., Mutimer, D.J. \& Bassendine, M.F. (1988) Primary biliary cirrhosis: identification of two major M2 mitochondrial autoantigens. Lancet, 1, 1067-1070.

Zhang, H., Carbone, M., Lleo, A. \& Invernizzi, P. (2015a) Geoepidemiology, Genetic and Environmental Risk Factors for PBC. Dig. Dis., 33, 94-101.

Zhang, X.X., Wang, L.F., Jin, L., Li, Y.Y., Hao, S.L., Shi, Y.C., Zeng, Q.L., Li, Z.W., Zhang, Z., Lau, G.K. \& Wang, F.S. (2015b) Primary biliary cirrhosis-associated hepatocellular carcinoma in Chinese patients: incidence and risk factors. World J. Gastroenterol., 21, 3554-3563. 